\title{
Draft genome sequence of multi-resistant Salmonella enterica subsp. enterica serovar Rissen strain 19CS0416 isolated from Vietnam reveals mcr-l plasmid mediated resistance to colistin already in 2013
}

Belen Gonzalez-Santamarina ${ }^{\circledR}$, Anne Busch ${ }^{1,3}$, Silvia Garcia-Soto ${ }^{1}$, Mostafa Y. Abdel-Glil1 ${ }^{1}$, Jörg Linde Reinhard Fries ${ }^{2}$, Diana Meemken ${ }^{2}$, Helmut Hotzel ${ }^{1}$, Herbert Tomaso ${ }^{1}$

1. Friedrich-Loeffler-Institut, Institute of Bacterial Infections and Zoonoses (IBIZ), Naumburger Str. 96a, 07743 Jena, Germany.

2. Institute of Food Safety and Food Hygiene, Section Meat Hygiene, Freie Universität Berlin, Königsweg 67, 14163 Berlin, Germany.

3. Department of Anesthesiology and Intensive Care Medicine, University Hospital Jena, Am Klinikum 1, 07747 Jena, Germany.

$\bowtie$ Corresponding author: Belen.gonzalezsantamarina@fli.de

(c) The author(s). This is an open access article distributed under the terms of the Creative Commons Attribution License (https://creativecommons.org/licenses/by/4.0/). See http://ivyspring.com/terms for full terms and conditions.

Received: 2019.12.06; Accepted: 2020.04.13; Published: 2020.07.03

\begin{abstract}
We report the first draft genome sequence of a Salmonella strain with plasmid-mediated resistance to colistin encoded by mcr-l gene in Vietnam. Salmonella enterica subsp. enterica serovar Rissen was isolated from a Vietnamese pig slaughterhouse in 2013. We can confirm that $\mathrm{mcr}-1$ gene is identical to the first reported mcr-l gene of the Escherichia coli strain SHP45, isolated in 2015 from a Chinese pig. The plasmid containing this gene in the strain 19 CS0416 was highly related ( $96.86 \%$ identity) to the plasmid (pHNSHP45) contained in this Chinese strain. Moreover, this plasmid was determined to be 100\% identical to a plasmid (p13P477T-7) belonging to an Escherichia coli (13P477T) found in Hong Kong during the same year in pigs. Our results will aid in understanding the dissemination of mcr-l gene in East Asia, dating back to as early as 2013.
\end{abstract}

Key words: Salmonella Rissen, multidrug resistance, colistin, genome sequence, pig.

\section{Text}

Salmonellosis, a foodborne disease caused by Salmonella spp., is of global concern due to the high number of infections and increasing resistance of the bacteria to antibiotics. This zoonotic pathogen occurs in many food-producing animals and can be transmitted by raw meat, eggs, and dairy products [1]. Non-typhoidal Salmonella are a major cause of food-borne gastroenteritis worldwide [2]. These strains can be host-restricted or host generalists, infecting a broad range of vertebrates [3]. Salmonella enterica subsp.enterica serovar Rissen strain is ubiquitous and the most common serovar in pig and human patients with diarrhea in Vietnam [4]. We analyzed a Salmonella enterica subsp. enterica serovar Rissen that was isolated from a pig carcass in 2013 in a
Vietnamese slaughterhouse. We determined the antibiotic susceptibility of isolate 19CS0416 and used next-generation sequencing data to analyze genes conferring antibiotic resistance, virulence genes, and to analize the plasmids.

Bacterial cultivation, species identification, and serotyping were performed using standard procedures according ISO 6579-1:2017 [5]. Antimicrobial susceptibility of the strain was assessed by determining the clinical minimum inhibitory concentration (MIC) breakpoints in accordance with the European Committee on Antimicrobial Susceptibility Testing [6] using VITEK 2 Compact system (bioMérieux, Marcy-l'Étoile, France). VITEK cards AST-N195 and AST-N248 were employed to 
determine MIC values $(\mathrm{mg} / \mathrm{l})$. The strain was categorized as resistant to ampicillin (MIC of $\geq 32$ $\mathrm{mg} / \mathrm{l}$ ), gentamicin (MIC of $\geq 16 \mathrm{mg} / \mathrm{l}$ ), tobramycin (MIC of $=8 \mathrm{mg} / \mathrm{l}$ ) and colistin (MIC of $\geq 16 \mathrm{mg} / \mathrm{l}$ ). The strain was categorized as susceptible (increased exposure) (new EUCAST nomenclature) [7] or intermediate (old definition) for: amoxicillin with clavulanic acid (MIC of $\geq 8 \mathrm{mg} / \mathrm{l}$ ) and tigecycline (MIC of $=2 \mathrm{mg} / \mathrm{l}$ ). The strain was categorized as susceptible (standard dosing regimen) to all other tested antibiotics piperacillin (MIC of $\leq 4 \mathrm{mg} / \mathrm{l}$ ), piperacillin-tazobactam (MIC of $\leq 4 \mathrm{mg} / \mathrm{l}$ ), cefalexin (MIC of $\leq 16 \mathrm{mg} / \mathrm{l}$ ), cefuroxime-axetil (MIC of $\leq 8$ $\mathrm{mg} / \mathrm{l}$ ), cefotaxime (MIC of $\leq 1 \mathrm{mg} / \mathrm{l}$ ), ceftazidime (MIC of $\leq 1 \mathrm{mg} / \mathrm{l}$ ), cefepime (MIC of $\leq 1 \mathrm{mg} / \mathrm{l}$ ), aztreonam (MIC of $\leq 1 \mathrm{mg} / \mathrm{l}$ ), ertapenem (MIC of $\leq 0.5$ $\mathrm{mg} / \mathrm{l}$ ), imipenem (MIC of $\leq 0.25 \mathrm{mg} / \mathrm{l}$ ), meropenem (MIC of $\leq 0.25 \mathrm{mg} / \mathrm{l}$ ), amikacin (MIC of $\leq 2 \mathrm{mg} / \mathrm{l}$ ), ciprofloxacin (MIC of $\leq 0.25 \mathrm{mg} / \mathrm{l}$ ), moxifloxacin (MIC of $\leq 0.25 \mathrm{mg} / \mathrm{l}$ ), fosfomycin (MIC of $\leq 16 \mathrm{mg} / \mathrm{l}$ ), trimethoprim (MIC of $\leq 0.5 \mathrm{mg} / \mathrm{l}$ ) and trimethoprim-sulfamethoxazole (MIC of $\leq 1 \mathrm{mg} / \mathrm{l}$ ).

For whole-genome sequencing, the strain was grown overnight at $37^{\circ} \mathrm{C}$ in $3 \mathrm{ml}$ of Luria-Bertani broth (Mast Diagnostica $\mathrm{GmbH}$, Reinfeld, Germany). DNA sequencing libraries were constructed using the Nextera XT Preparation Kit (Illumina Inc., San Diego, CA, USA) in accordance with the manufacturer's instructions. Paired-end sequencing was performed on the Illumina MiSeq platform (Illumina Inc., San Diego, CA, USA) using a 300-cycle MiSeq reagent kit. In total, 2,351,952 reads were generated. A quality check was performed with FastQC v0.11.5 (available at http://www.bioinformatics.babraham.ac.uk/ projects/fastqc/). Reads were de novo assembled using SPAdes v3.12.0 in Bayes Hammer mode (--careful) [8] and evaluated with QUAST v4.3 [9] in standard settings. The filtering of the sample was performed by removing contigs with coverage less than $5 \times$ and size below 500 bases. The filtered genome assembly was represented by 65 contigs with an N50 contig length of $554,575 \mathrm{bp}$ in which the largest contig had $810,994 \mathrm{bp}$. The average coverage was 116-fold. The combined length of the contigs was 5,083,583 bp with a GC content of $51.92 \%$. Annotation was performed with RAST Server (v2.0) [10]. Annotation features include 5.203 coding sequences (CDS), 10 rRNAs, 79 tRNAs, and 1 transfer-messenger RNA (tmRNA).

Data were submitted and analyzed using an in-house Linux-based bioinformatics pipeline WGSBAC (available at https://gitlab.com/ FLI_Bioinfo/WGSBAC). Genes coding for resistance were detected using abricate v08.13 (available at https://github.com/tseemann/abricate). Genotyping was performed with MLST (v2.16.1) showing a sequence type 469 (aroC (92), dna $\mathrm{N}(107)$, hemD (79), hisD (156), purE (64), sucA (151) and thrA (87)) and the in silico serotyping detection was performed with SISTR (v1. 0.2) [11] confirming the serovar Rissen with antigenic formula 6,7,14:f,g:-. Data were analyzed against the databases ResFinder [12] and CARD [13]. The databases predicted antimicrobial resistance to penicillins and first-generation cephalosphorins, aminoglycosides, fluoroquinolones, colistin, and lincosamides (bla $a_{\mathrm{TEM}-1 \mathrm{~b},}$ aac (6')-Iaa, aac(3)-IId, ant(3")-Ia, $m d f(\mathrm{~A})$, floR, tet(A), tet(M), mcr-1, $\ln u(\mathrm{~F}))$ (Table S1). Using abricate against the PlasmidFinder [14] database, genomic regions (replicons) of five independent plasmids could be identified (IncI2_1_Delta, IncFIC (FII)_1, ColRNAI_1, Col156_1, Col (BS512)) (Table S2). Plasmid preparation was performed with the QIAGEN® Plasmid Mini Kit (QIAGEN GmbH, Hilden, Germany) according to the manufacturer's instructions. We could confirm the presence of three plasmids in agarose gel electrophoresis [15, 16]. One hundred and thirty-seven virulence genes (Table S3) were predicted using abricate and the Virulence Factors Database [17]. Salmonella pathogenicity islands (SPIs) are suspected to be acquired by horizontal gene transfer and to have an effect on the structure of the genome $[18,19]$. SPIFinder [20] confirmed three Salmonella pathogenicity islands (SPI-2, SPI-8 and C63PI) (Table S4). This is below the average (1-14 SPIs) and may be associated with individual serovar Rissen isolates as described before [20]. Therefore, using the virulence genes output predicted by the Virulence Factors Database, we have looked for the virulence genes associated with another SPI [21]. Genes related with SPI-I, SPI-3 and SPI-5 were found (Table S4) but not SPI-4. To confirm the presence or absence of SPI-4 the specific pathogenicity island SPI-4 genomic sequence of S. Typhimurium with accession number KP234070 was downloaded and mapped against our sequence 19CS0416 using Geneious prime (version 11.1.5) (Biomatters, Ltd., Auckland, 1010, New Zealand). The six genes siiABCDEF [22] present in this region were found in two different contigs of the studied sequence (Table S4). We could confirm that 7 SPIs were present in this strain (SPI-1, SPI-2, SPI-3, SPI-4, SPI-5, SPI-8 and C63PI).

For the evaluation of the mor-1 gene, the program Geneious was used to find this gene in the annotated contigs. It was found in contig number 18 . The $m c r-1$ gene (annotated as Lipid A phosphoethanolamine transferase by RAST server) was extracted and aligned against the mcr-1 gene of the Escherichia (E.) coli strain SHP45 plasmid pHNSHP45 published by Liu et al. in 2016. The alignment showed 100\% 
identity with published sequence [23]. In the same contig, a replication initiation protein and genes related to Inc1 plasmid were found (Table S5). The sequence has $96.82 \%$ identity with the E. coli strain SHP45 plasmid pHNSHP45 (accession number KP347127.1). Using BLASTn [24] (https://blast.ncbi.nlm.nih.gov/Blast.cgi) it has been found that our strain has a $100 \%$ identity with the $E$. coli strain 13P477T plasmid p13P477T-7 (accession number NZ_CP021103.1), which belongs to a strain published by Ho et al. in 2017 taken from pig feces in Hong Kong in 2013.

Recombination and rearrangement events are driving factors for evolution, and the varying content of plasmids, AMR genes, and SPIs describes the diversity within a species. In further studies, the $m c r-1$ gene-containing genomes will be studied for their genomic evolution in a cross-species approach to elucidate the recombination and rearrangement events on the aforementioned genomic features.

The emergence of plasmid-mediated colistin resistance gene $m c r-1$ was first reported in 2015 in an E. coli strain isolated in a pig in China [23]. Since then, the gene was found in more than 30 countries [25]. In retrospect, the $m c r-1$ gene was also reported in E. coli isolates from China in the 1980ies [26], as well as in Salmonella isolates collected in Germany in 2008 [27]. In Vietnam, the first detected $m c r-1$ gene reported in E. coli was detected by PCR in 2013 in poultry [28] and a year later in pigs [29] (Fig. 1). In Salmonella spp., the first phenotypically colistin-resistant isolate was found in 2012 in pigs [30], and the first $m c r-1$ gene detection in this genus was confirmed by PCR in an isolate from pork between 2016 and 2017 (Fig. 1). Here we report the first draft genome sequence of a Salmonella enterica subsp. enterica serovar Rissen strain with a plasmid-mediated resistance to colistin encoded by the mcr-1 gene from Vietnam, which is identical to the first reported colistin-mediated $\mathrm{mcr}-1$ gene. The plasmid was closely related $(96.86 \%$ identity) to the plasmid containing mcr-1 (pHNSHP45) in the Chinese strain and it showed $100 \%$ identity to plasmid p13P477T-7 belonging to an E. coli (13P477T) found in Hong Kong in the same year in pigs. Our results will aid in understanding the dissemination of this gene and plasmid in East Asia, dating back to as early as 2013 and in different species as compared to the first reported mor-1 carrying strain. The results show the importance of the One Health Approach for the surveillance of resistance in bacteria in food-producing animals in the food chain, food products, humans and the environment.

\section{Nucleotide sequence accession number}

Whole-genome sequence of multi-resistant Salmonella enterica subsp. enterica serovar Rissen strain 19CS0416 was submitted to SRA database with the accession number: PRJNA591084, BioProject PRJNA591084 (https:/ / www.ncbi.nlm.nih.gov/biopr oject/PRJNA591084), and BioSample SAMN 13381784 (https:/ / www.ncbi.nlm.nih.gov/biosample /13381784).

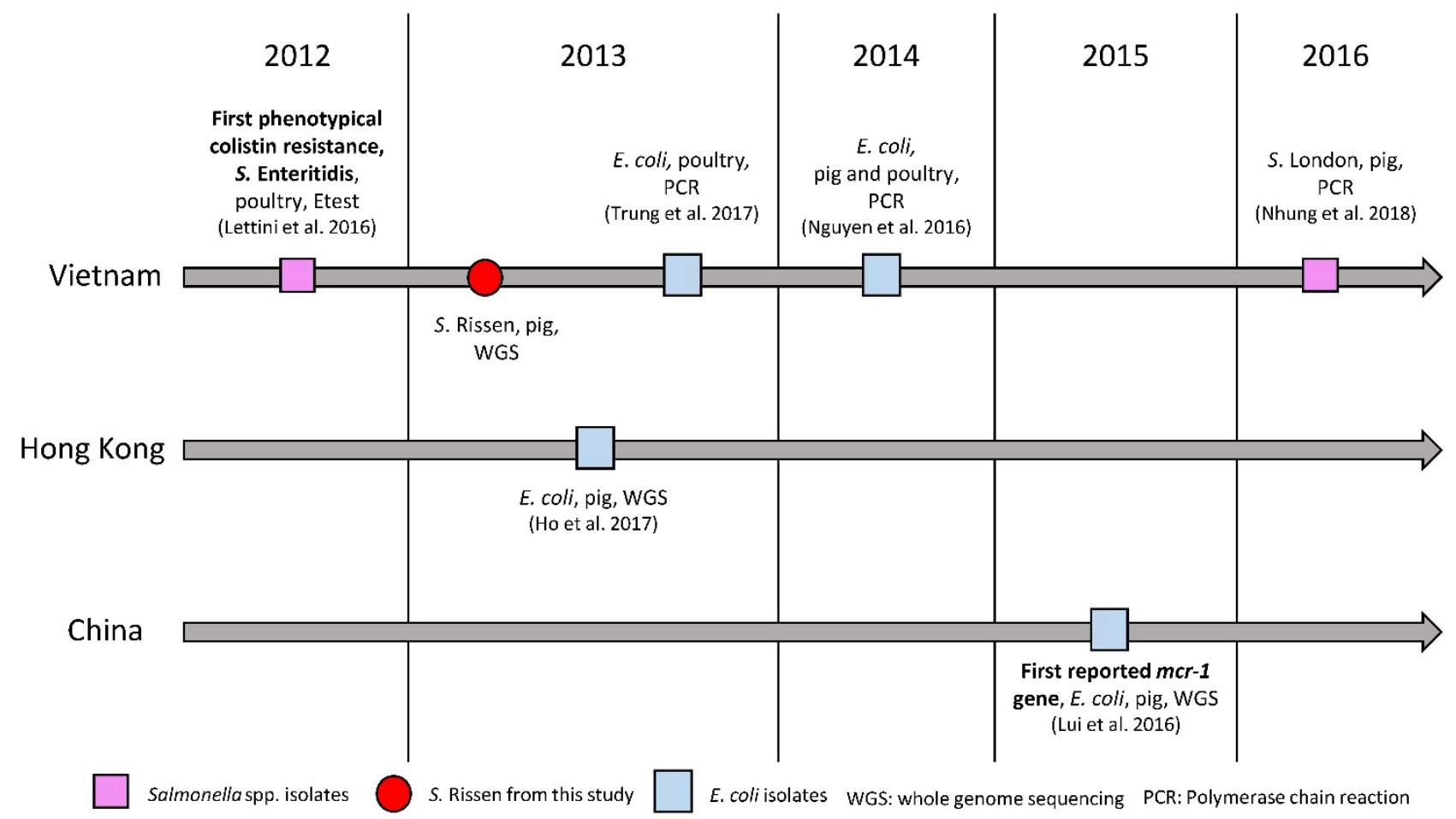

Figure 1. Colistin resistance and mcr-I gene in Vietnam, Hong Kong and China. 


\section{Supplementary Material}

Supplementary figures and tables. http://www.jgenomics.com/v08p0076s1.pdf

\section{Acknowledgments}

We thank Fiona Balzer and Herlinde Irsigler for skillful technical assistance. For this work, Belén González Santamarina, Silvia Garcia Soto, and Mostafa Y. Abdel-Glil were supported by in-house projects of the Friedrich-Loeffler-Institut on "AMR-One Health". Anne Busch was supported by a grant of the German Federal Ministry of Education and Research within the framework of the project Ess-B.A.R. (FKZ 13N13983) and by the Deutsche Forschungsgemeinschaft (DFG, German Research Foundation) under Germany's Excellence Strategy EXC 2051 - Project-ID 390713860.

\section{Competing Interests}

The authors have declared that no competing interest exists.

\section{References}

1. Forshell LP, Wierup M. Salmonella contamination: a significant challenge to the global marketing of animal food products. Rev Sci Tech Oie. 2006; 25: 541-54.

2. Majowicz SE, Musto J, Scallan E, Angulo FJ, Kirk M, O'Brien SJ, et al. The global burden of nontyphoidal Salmonella gastroenteritis. Clin Infect Dis. 2010; 50: 882-9.

3. Connor TR, Owen SV, Langridge G, Connell S, Nair S, Reuter S, et al. What's in a Name? Species-Wide Whole-Genome Sequencing Resolves Invasive and Noninvasive Lineages of Salmonella enterica Serotype Paratyphi B. MBio. 2016; 7.

4. Nhung NT, Van NTB, Cuong NV, Duong TTQ, Nhat TT, Hang TTT, et al. Antimicrobial residues and resistance against critically important antimicrobials in non-typhoidal Salmonella from meat sold at wet markets and supermarkets in Vietnam. International Journal of Food Microbiology. 2018; 266: 301-9.

5. Xuan SD, Fries R, Meeyam T. Quantifying Salmonella spp in slaughterhouse and pork market associated with human health in Hung Yen, Vietnam. CGIAR. 2013.

6. [Internet] EUCAST. Breakpoint tables for interpretation of MICs and zone diameters. Version 9.0. 2019; http:/ / www.eucast.org.

7. [Internet] EUCAST. Redefining susceptibility testing categories S, I and R. 2019 http://www.eucast.org/fileadmin/src/media/PDFs/ EUCAST_files/EUCAST_Presentations/2018/EUCAST_-_Intermediate _category_-_information_for_all.pdf. 2019.

8. Bankevich A, Nurk S, Antipov D, Gurevich AA, Dvorkin M, Kulikov AS, et al. SPAdes: a new genome assembly algorithm and its applications to single-cell sequencing. J Comput Biol. 2012; 19: 455-77.

9. Gurevich A, Saveliev V, Vyahhi N, Tesler G. QUAST: quality assessment tool for genome assemblies. Bioinformatics (Oxford, England). 2013; 29.

10. Aziz RK, Bartels D, Best AA, DeJongh M, Disz T, Edwards RA, et al. The RAST server: Rapid annotations using subsystems technology. Bmc Genomics. 2008; 9

11. Yoshida CE, Kruczkiewicz P, Laing CR, Lingohr EJ, Gannon VP, Nash $\mathrm{JH}$, et al. The Salmonella In Silico Typing Resource (SISTR): An Open Web-Accessible Tool for Rapidly Typing and Subtyping Draft Salmonella Genome Assemblies. PLoS One. 2016; 11: e0147101.

12. Zankari E, Hasman H, Cosentino S, Vestergaard M, Rasmussen S, Lund $\mathrm{O}$, et al. Identification of acquired antimicrobial resistance genes. J Antimicrob Chemoth. 2012; 67: 2640-4.

13. Jia BF, Raphenya AR, Alcock B, Waglechner N, Guo PY, Tsang KK, et al. CARD 2017: expansion and model-centric curation of the comprehensive antibiotic resistance database. Nucleic Acids Research. 2017; 45: D566-D73.
14. Carattoli A, Zankari E, Garcia-Fernandez A, Voldby Larsen M, Lund O, Villa L, et al. In silico detection and typing of plasmids using PlasmidFinder and plasmid multilocus sequence typing. Antimicrob Agents Chemother. 2014; 58: 3895-903.

15. Becker L, Steglich M, Fuchs S, Werner G, Nubel U. Comparison of six commercial kits to extract bacterial chromosome and plasmid DNA for MiSeq sequencing. Sci Rep. 2016; 6: 28063.

16. Hanel I, Hotzel H, Tomaso H, Busch A. Antimicrobial Susceptibility and Genomic Structure of Arcobacter skirrowii Isolates. Frontiers in microbiology. 2018; 9: 3067.

17. Liu B, Zheng DD, Jin Q, Chen LH, Yang J. VFDB 2019: a comparative pathogenomic platform with an interactive web interface. Nucleic Acids Research. 2019; 47: D687-D92.

18. Juhas M, van der Meer JR, Gaillard M, Harding RM, Hood DW, Crook DW. Genomic islands: tools of bacterial horizontal gene transfer and evolution. FEMS Microbiol Rev. 2009; 33: 376-93.

19. Garcia-Vallve S, Romeu A, Palau J. Horizontal gene transfer in bacterial and archaeal complete genomes. Genome research. 2000; 10: 1719-25.

20. Roer L, Hendriksen RS, Leekitcharoenphon P, Lukjancenko O, Kaas RS, Hasman $\mathrm{H}$, et al. Is the Evolution of Salmonella enterica subsp. enterica Linked to Restriction-Modification Systems? mSystems. 2016; 1.

21. Marcus SL, Brumell JH, Pfeifer CG, Finlay BB. Salmonella pathogenicity islands: big virulence in small packages. Microbes Infect. 2000; 2: 145-56.

22. Kiss T, Morgan E, Nagy G. Contribution of SPI-4 genes to the virulence of Salmonella enterica. Fems Microbiol Lett. 2007; 275: 153-9.

23. Liu YY, Wang Y, Walsh TR, Yi LX, Zhang R, Spencer J, et al. Emergence of plasmid-mediated colistin resistance mechanism MCR-1 in animals and human beings in China: a microbiological and molecular biological study. Lancet Infect Dis. 2016; 16: 161-8.

24. Coordinators NR. Database resources of the National Center for Biotechnology Information. Nucleic Acids Res. 2013; 41: D8-D20.

25. Lima T, Domingues S, Da Silva GJ. Plasmid-Mediated Colistin Resistance in Salmonella enterica: A Review. Microorganisms. 2019; 7.

26. Shen ZQ, Wang Y, Shen YB, Shen JZ, Wu CM. Early emergence of mcr-1 in Escherichia coli from food-producing animals. Lancet Infect Dis. 2016; 16: 293-.

27. Borowiak M, Hammerl JA, Fischer J, Szabo I, Malorny B. Complete Genome Sequence of Salmonella enterica subsp. enterica Serovar Paratyphi B Sequence Type 28 Harboring mcr-1. Genome Announc. 2017; 5 .

28. Trung NV, Matamoros S, Carrique-Mas JJ, Nghia NH, Nhung NT, Chieu TTB, et al. Zoonotic Transmission of mcr-1 Colistin Resistance Gene from Small-Scale Poultry Farms, Vietnam. Emerg Infect Dis. 2017; 23: 529-32.

29. Nguyen NT, Nguyen HM, Nguyen CV, Nguyen TV, Nguyen MT, Thai HQ, et al. Use of Colistin and Other Critical Antimicrobials on Pig and Chicken Farms in Southern Vietnam and Its Association with Resistance in Commensal Escherichia coli Bacteria. Appl Environ Microb. 2016; 82: 3727-35.

30. Lettini AA, Than TV, Marafin E, Longo A, Antonello K, Zavagnin P, et al. Distribution of Salmonella Serovars and Antimicrobial Susceptibility from Poultry and Swine Farms in Central Vietnam. Zoonoses Public Hlth. 2016; 63: 569-76. 